\title{
Epidural Hematoma: A Systematic Review
}

\author{
Vítor Ferraz Silva Tacconi, ${ }^{1}$ Luciana Karla Viana Barroso, ${ }^{2}$ Nicole Bruna da Costa Azevedo, ${ }^{3}$ Tayná Rodrigues de Souza, ${ }^{3}$ Cíntia \\ Thaís Duarte Matias ${ }^{3}$ \\ 'Departament of Medicine, Universidade Potiguar - UNP, Parnamirim, RN, Brazil \\ ${ }^{2}$ Teacher of Neuroanatomia Funcional of Centro Universitário UNIFACISA and the Universidade Federal de Campina Grande - UFCG, Catolé, \\ Campina Grande, PB, Brazil \\ ${ }^{3}$ Departament of Medicine, Centro Universitário - UNIFACISA, Campina Grande, PB, Brazil
}

Disclose and conflicts of interest: none to be declared by all authors

\begin{abstract}
Introduction: epidural hematoma $(E D H)$ is an event that can result in mortality and morbidity. Understanding it is critical to a fast diagnosis that enables successful management. In the perspective, this article aim to analyze the physiopathology of epidural hematoma. Specifically, the proposal develops a survey on the literature that covers the topic of epidural hematoma, to update and review in case reports the neurological damage and to seek the new concepts relative to the surgical practice, using the method of bibliographic study. Besides to bring some epidemiological data about this theme in Brazil in recent years. The results showed that the risks involved in the occurrence of an EDH go far beyond physical impacts, tumor lesions and infections, extending to cases of hemorrhage due to the use of drugs. Based on the DataSUS, there are no significant variations in hospitalizations due to intracranial hemorrhage or intracranial trauma in recent years. For treatment, minimally invasive surgery is one of the best alternatives to treat patients with EDH due to small incisions, reduced surgical time, reduced risk of anesthesia and avoid craniotomy. It is concluded that epidural hematoma requires an early diagnosis so that surgical intervention prevents other avoidable complications from occurring. Besides that, the public health system needs to have adequate structure, effective prevention strategies and improved EDH care.
\end{abstract}

Key words: Epidural Hematoma; Nontraumatic Epidural Hemorrhage; Intracranial Trauma; Intracranial Hemorrhage.

\section{Introduction}

According to Ahn and Proctor (2019), ${ }_{1}$ epidural hematoma $(\mathrm{EDH})$ is a hemorrhage in the space between calvaria and dura mater, and is almost always the result of trauma. Moreover, EDH may also be called an extradural hematoma. Removal of the dura mater from the skull is caused due to the mass effect on the brain the proportion that the hematoma grows resulting in mortality and morbidity. Successful management of epidural hematoma depends on immediate diagnosis.

These same authors describe that, in general, the suture lines of the skull are not crossed in the epidural hematoma, because the dura mater is less adherent to the skull in the center of the bone plate, which is the area in which it occurs the resorption and remodeling of the bone and, therefore, is more adherent in the suture lines, where the osteoblastic activity is more intense.

The venous sinuses of the dura and the middle meningeal arteries are usually cut during the associated fractures, which are mostly linear. In addition, blood usually accumulates on both sides of the bone adjacent to the fracture. Therefore, EDH is frequently associated with subgaleal hematoma (Ahn; Proctor, 2019). ${ }^{1}$

Understanding epidural hematoma is crucial with regard to its consequences, so immediate diagnosis is extremely important for the successful management of epidural hematoma. According to McBride (2019), ${ }^{2}$ patient death or irreversible damage to brain tissue caused by elevated intracranial pressure, cerebral herniation and expansive hematoma are acute symptoms of EDH, which is considered a neurological emergency requiring frequent surgical treatment. However, some selected patients with EDH with small volume, good clinical presentation and who remain stable can be treated with non-surgical procedure.

From this perspective, this article aims to analyze the physiopathology epidural hematoma. Specifically, the proposal develops a survey on the literature that covers the topic of epidural hematoma, to update and review in case reports the neurological damages and searching for the new concepts relative to the surgical practice. In addition to bring some epidemiological data about this theme in Brazil in recent years.

\section{Materials and Methods}

The present article is a bibliographic study that has a character of literature review carried out between the months of january to august of 2019. To do so, a systematic search was made for published articles, in the last 5 years, with the filters for free full text, research in humans and the best match articles were used for the PubMed. Other journals indexed in the Scielo, LILACS, Google Scholar, Cochrane Library, Capes and Bireme databases were also used in the research using the 
free-access full-text filter. Complete texts paid on the Up to Date platform on the topic Epidural Hematoma were also used in the research. As well as researches were developed on the DataSUS page for the collection of secondary data related to the epidemiology associated with intracranial hemorrhage, intracranial trauma and nontraumatic epidural hemorrhage.

The elaboration of table 1 was developed from the survey on the Department of Disease Surveillance and noncommunicable aggravate and Health Promotion (DANTPS/SVS/MS) platform, accessing the "Content Centers" and choosing the "Monitoring Panel", then accessing the "Mortality Monitoring Panel", and finally selecting the item "Mortality Monitoring Panel CID - 10", available at: http://svs.aids.gov.br/dantps/ centrais-de-conteudos/paineis-de-monitoramento/ mortalidade/cid10/.

Table 1. Deaths from nontraumatic epidural hemorrhage in Brazil per year place of registration: deaths by occurrence - ICD-10 category: nontraumatic epidural hemorrhage - $162.1^{7}$

\begin{tabular}{l|c|c|c|c|c}
\hline \multicolumn{7}{c}{ Periods } \\
\hline \multicolumn{1}{c|}{ Region } & $\mathbf{2 0 1 5}$ & $\mathbf{2 0 1 6}$ & $\mathbf{2 0 1 7}$ & $\mathbf{2 0 1 8 *}$ & 2019* \\
\hline North & 1 & 0 & 0 & 1 & 0 \\
\hline Northeast & 2 & 3 & 3 & 5 & 0 \\
\hline Southeast & 8 & 3 & 5 & 16 & 2 \\
\hline South & 3 & 3 & 7 & 1 & 0 \\
\hline Midwest & 1 & 2 & 0 & 1 & 0 \\
\hline Total & 15 & 11 & 15 & 24 & 2 \\
\hline
\end{tabular}

Years presented with * are preliminary

Reference: Ministry of Health. Department of Disease Surveillance and noncommunicable aggravate and Health Promotion - DANTPS/SVS/MS ${ }^{7}$

Tables 2 and 3 were made with the data found in the Hospital Information System - SIH/SUS platform, accessing the DATASUS website, in the tab "Epidemiological Information and Morbidity", then accessing "Hospital Morbidity of SUS (SIH/SUS)", in the item "General, by place of hospitalization - from 2008", then also selecting the geographic coverage "Brazil by Region and Federation Unity". This information is available at: http://tabnet.datasus.gov.br/cgi/ deftohtm.exe?sih/cnv/niuf.def.

The descriptors used for the search followed the description of the Health Sciences Descriptors (DeCS) ${ }^{3}$ terms, the keywords were combined using the Boolean operators OR and AND, without linguistic restriction using the following words: "Epidural Hematoma", "Intracranial Trauma", "Intracranial Hemorrhage" and "Nontraumatic Epidural Hemorrhage" for national and international articles.

All the articles obtained in the databases through the research suffered the application of inclusion and exclusion criteria. The first phase of the review consisted of analyzing the titles and abstracts of the articles related to the topic, which were identified and evaluated, to select those that met the eligibility criteria, such as: been published in the last 5 years, have in their title and/or in their abstract some of the descriptors used in the searches to the databases. Articles that presented repeated information, duplicated or that the topic addressed was not related to the research proposal were excluded. The studies that were selected as relevant and that generated doubts were retained for further analysis of the text in its entirety.

The second phase of the review consisted of the extraction of data from the studies selected by five authors. All eligible records were read in full and data available in the text were extracted. We excluded those who, despite appearing in the search result, did not approach the subject adequately with the variables of interest in this study.

\section{Results and Discussion}

\section{Physiopathology}

According Ren et al. (2018), ${ }^{4}$ postoperative epidural hematoma (POEDHs) is characterized by some computed tomography findings like hyperdense collection under the skull, hematoma with format biconvex lens-shaped and aspect homogeneous. Moreover, most POEDHs are found within 3 days after the surgery.

Table 2. Hospital Morbidity of SUS - by place of hospitalization in Brazil -Morbidity List CID -10: Intracranial hemorrhage ${ }^{11}$

\begin{tabular}{|c|c|c|c|c|c|c|c|}
\hline \multicolumn{8}{|c|}{ Periods } \\
\hline \multicolumn{2}{|c|}{2016} & \multicolumn{2}{|c|}{2017} & \multicolumn{2}{|r|}{2018} & \multicolumn{2}{|c|}{ 2019/ until may } \\
\hline Region & hospitalization & Region & hospitalization & Region & hospitalization & Region & hospitalization \\
\hline North & 1.638 & North & 1.660 & North & 1.674 & North & 137 \\
\hline Northeast & 5.994 & Northeast & 5.967 & Northeast & 6.051 & Northeast & 450 \\
\hline Southeast & 12.298 & Southeast & 12.302 & Southeast & 12.725 & Southeast & 1.103 \\
\hline South & 5.411 & South & 5.354 & South & 5.014 & South & 428 \\
\hline Midwest & 2.152 & Midwest & 1.931 & Midwest & 1.919 & Midwest & 147 \\
\hline Total & 27.493 & Total & 27.214 & Total & 27.383 & Total & 2.265 \\
\hline
\end{tabular}

Reference: Ministry of Health. Hospital Information System - SIH/SUS ${ }^{11}$ 
Table 3. Hospital Morbidity of SUS - by place of hospitalization in Brazil - Morbidity List CID - 10: Intracranial trauma ${ }^{11}$

PERIODS

\begin{tabular}{c|c|c|c|c|c|c|c}
\hline \multicolumn{2}{l|}{2016} & \multicolumn{2}{c|}{2017} & \multicolumn{2}{c}{ 2018 } & \multicolumn{2}{c}{ 2019/ until may } \\
\hline Region & hospitalization & Region & hospitalization & Region & hospitalization & Region & hospitalization \\
\hline North & 8.559 & North & 8.249 & North & 7.646 & North & 376 \\
\hline Northeast & 29.016 & Northeast & 28.015 & Northeast & 27.741 & Northeast & 1.870 \\
\hline Southeast & 43.811 & Southeast & 42.146 & Southeast & 41.159 & Southeast & 3.567 \\
\hline South & 17.877 & South & 19.206 & South & 17.717 & South & 1.446 \\
\hline Midwest & 7.234 & Midwest & 7.243 & Midwest & 7.264 & Midwest & 538 \\
\hline Total & 106.497 & Total & 104.859 & Total & 101.527 & Total & 7.797 \\
\hline
\end{tabular}

Reference: Ministry of Health. Hospital Information System - SIH/SUS ${ }^{11}$

In an acute EDH (Epidural Hematoma) some findings on CT are classic, like: Extra-axial mass, biconvex or lenticular format, homogeneous aspect and hyperdense (60-90 HU). Hyperacute EDH is typically observed a heterogeneous or mixed CT pattern of hyperdensity and hypodensity. These different densities represent a mixture of liquid blood and clotted. Moreover, the hyperdensity can represent the solid state of clotted blood and may increase during the first 3 days. (REN et al., 2018). ${ }^{4}$ An example of epidural hematoma can be seen in Figure 1.
2015 to May 2019. This indicates that doctors should always be aware of this possibility of diagnosis even without trauma, as it is a condition that can be fatal.

From 2015 until May 2019, the region of Brazil that accumulated the most deaths due to non-traumatic epidural hemorrhages was the Southeast with a total of 34 deaths, followed by the South with 14 cases. The region with the lowest number of cases in this same time interval was the Midwest. However, it is noteworthy that the information regarding the 2018 and 2019 data are preliminary and may change.
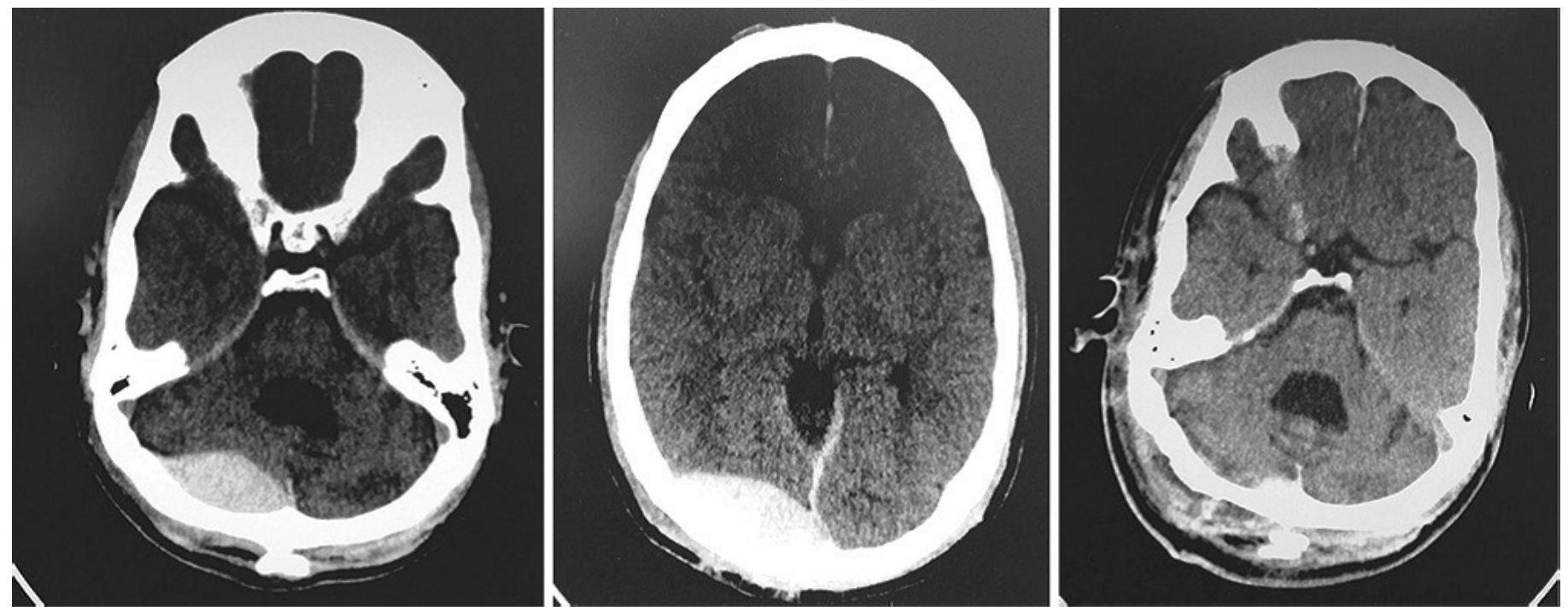

Figure 1. (a) A patient with posterior fossa extradural hematoma straddling the transverse sinus showing the infratentorial part, (b) supratentorial part of extradural hematoma in the same patient as in Figure $2 a$, (c) postoperative scan of patient in Figure 2a showing complete evacuation. (Reference: Verma et al., 2018: 228). ${ }^{5}$

The risks involved with the occurrences of an EDH go far beyond physical impacts, tumor lesions and infections, extending to cases of hemorrhage resulting from the use of medications. A case report of a 39-year-old woman at the Instituto de Neurologia de Curitiba (INC) demonstrates the risk associated with anticoagulants and the EDH. In the study it was reported that the active principle Rivaroxabana caused a secondary spontaneous epidural hematoma in the patient. (RUSCHEL et al., 2016). ${ }^{6}$

According to Table 1, non-traumatic epidural hemorrhages are the cause of death in 67 people from
Chen et al. (2018) ${ }^{8}$ in your study described that the prognosis of EDH is excellent if early diagnosis is made in conjunction with a surgical decompression. Besides, the authors highlight that spontaneous EDH is a rare condition, which may have some unusual triggers such as crying and hyperventilation, the presence of headaches with signs of increased intracranial pressure preceded by hyperventilation suggests that computed tomography examination should be done.

According Fang et al. (2018) ${ }^{9}$ a poor prognosis and permanent injury can be associate an inappropriate management of spinal epidural hematoma (SEH), 
being this pathology an emergent complication. Figure 2 shows an EDH that extends from the level of the second cervical vertebra to the sixth cervical vertebra.

Fang et al. (2018) ${ }^{9}$ suggest that exploratory surgery is a good option in patients who incurs spinal cord injury during vertebroplasty, because neurological deficits occurs secondary to acute spinal epidural hematoma (SEH). Moreover, theses authors affirm that neurological recovery is multifactorial, and the key for the postsurgical success is the timing of surgery, and timely decompression and transfer surgery have a special function to shorten the time of patient recovery. hemorrhage, as well as the risk of other hematomas, such as the subarachnoid hematoma and the subdural hematoma.

Table 3 shows the number of hospitalizations in Brazil due to Intracranial trauma. According to table 3 , the hospitalizations were over 100 thousand cases per year in the last 3 consecutive years, in addition the highest concentration of these cases occur in the Southeast and Northeast. Cranial trauma is one of the major causes of epidural hematoma, and it is important to emphasize that these events can cause deaths.
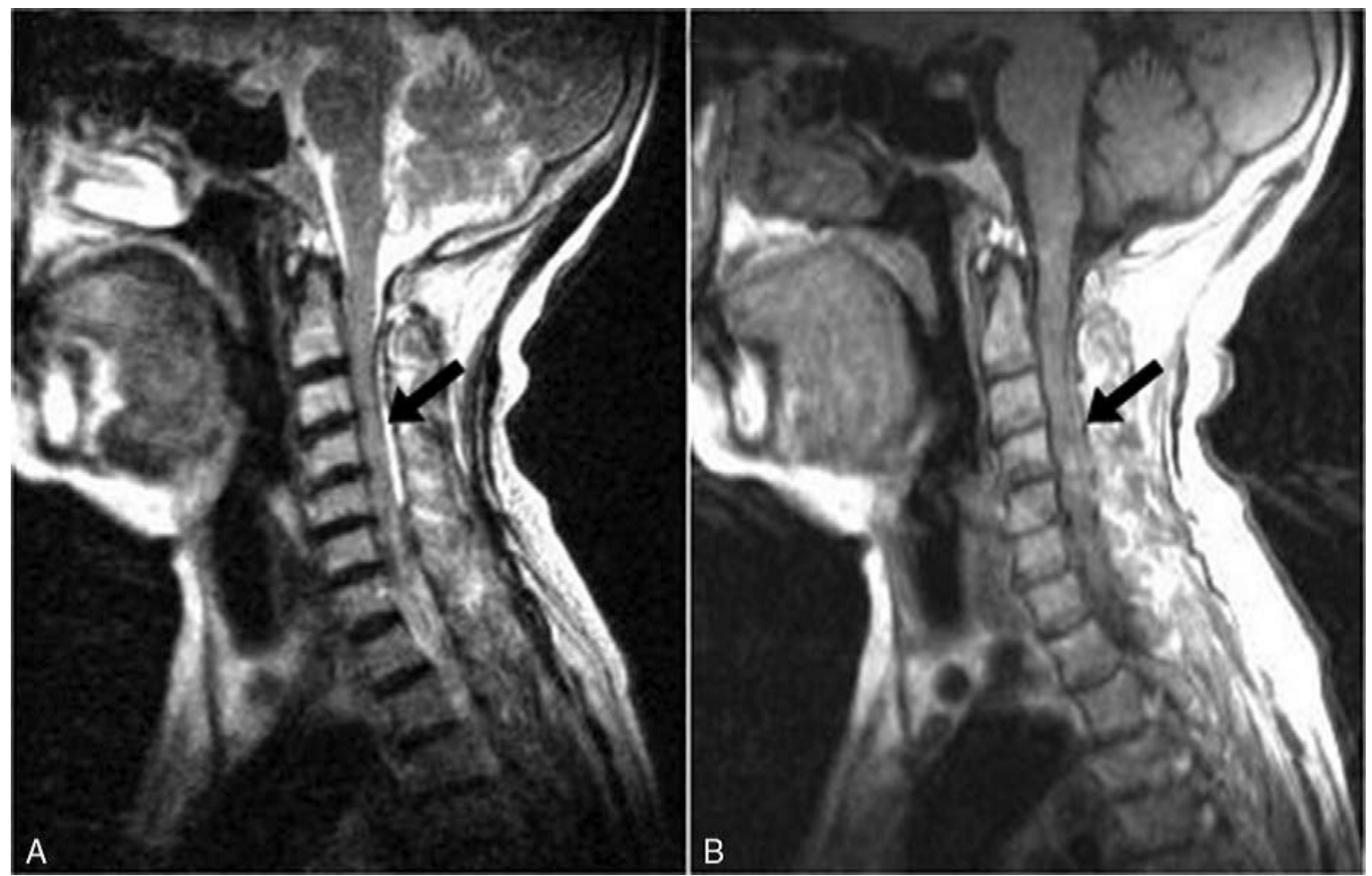

Figure 2. Cervical magnetic resonance imaging (MRl;sagittal) showed a longitudinal dorsal epidural hematoma (A: T2-weighted, B: T1-weighted) extending from C2 to C6 level. (Reference: Li et al. 2017: 2)10

\section{Epidemiology}

In relation to the morbidity of intracranial hemorrhages and cranial trauma of the years 2016, 2017, 2018 and 2019 (until may) can be visualized in Tables 2 and 3.

As Table 2 shows, there were no significant changes in hospitalizations for intracranial hemorrhage in the last 3 consecutive years. However, this morbidity is a worrying cause of hospitalization in Brazil, with epidural hematoma being one of the components that integrate the intracranial hemorrhage. According to these data, in the last years the southeast region was the region with the highest number of cases of hospitalization for this type of hemorrhage, followed by the northeast region, in the second place, with a large number of cases per year as well.

These data reveal the seriousness of this situation and alert not only to the risk of death due to epidural hematoma, which constitutes intracranial

\section{Treatment}

Epidural hematoma is a case of medical emergency and, due to its importance, several studies have been carried out seeking the use of new less invasive therapies or even the improvement of conservative techniques already used for many years.

Factors such as size and location of hematomas are important in determining the procedure to be performed, avoiding possible more serious neurological impairments.

According Rosi Junior et al. (2015), ${ }^{12}$ large and smalls hematomas carry a risk of cause irreversible damage to the brain stem or compressing structures. Theses authors affirm that Smalls EDHs should be operated on if they are located in the posterior or middle fossa, and the large EDHs must be operated on, regardless of their location. The most used surgical technique in these cases is the craniotomy. The time until surgery is 
performed is essential and should be reduced, because there is strong evidence that a delay in excising the EDH may compromise prognosis.

This type of hematoma may arise as a consequence of a Craniocerebral Trauma, but may also be secondary to some surgical procedure that may offer vascular compromise.

Moreira, Adry and Pereira (2017) $)^{13}$ used the example of decompressive craniectomy to express the surgical procedure as a resolving method for such events, being more used in the treatment of intracranial hypertension refractory due to severe brain injury, however, this procedure can cause serious complications such as the contralateral epidural hematoma. In such cases, the recognition of this lesion in a timely manner may influence the early accomplishment of the postoperative $\mathrm{CT}$ scans and, when diagnosed the presence of $\mathrm{EDH}$, immediate intervention will promote better results. Unexplained intraoperative cerebral edema or the presence of pupillary abnormalities in the postoperative period stand out as a good indicator for the development of the contralateral hematoma.

Bulstrode et al. (2017) ${ }^{14}$ emphasized the importance of a rapid and efficient approach for the decompression of an epidural hematoma before definitive neurosurgical management, obtaining a significant improvement in the prognosis. In obtunded patients with Computed Tomography-confirmed epidural hematoma, they propose temporizing by craniostomy and partial drainage of the collection using intraosseous needle, while preparations are made for transfer. They describe the application of this technique with a practical example of the beginning of the procedure in the anesthesia room while the patient is being stabilized for emergency craniotomy.

Epidural hematoma may still be a consequence of another surgical procedure such as cranioplasty, used to reconstruct cranial defects in patients after decompressive craniectomy. According to Peng et al. $(2018)^{15}$ repeated surgery compromises patient safety and delays recovery, so fibrinolytic therapy can be considered an optional treatment for postoperative epidural hematoma associated with cranioplasty, especially in patients who refused further operative treatment or who are not optimal candidates for a second surgery. They demonstrated that the use of this temporary catheter for the first operation, along with injecting urokinase (UK) through the subgaleal drain catheter, increased the hematoma drainage rate. UK could dissolve fibrin and fibrinogen and convert plasminogen into plasmin; therefore, UK may be a good candidate for dissolving hematomas. The major disadvantage of UK is the increased risk of rebleeding. Therefore, choosing the appropriate dose of UK may be important in preventing rebleeding. However, in our hands, this procedure helped avoid a second operation, along with the associated complications.

According Ren et al. (2018) $)^{4}$ smalls POEDHs and large EDH can be treated by different ways. Patients with small POEDHs are often asymptomatic or present with only headaches or minor neurologic deficits and can be treated conservatively. On other hand, the large EDH can be a life threat, because this hematoma can show a mass effect and induce a brain herniation, so the emergency evacuation is necessary.

In Figure 3 and 4 it is possible to verify the presence of $\mathrm{EDH}$, and the minimally invasive maneuver used in the patient.
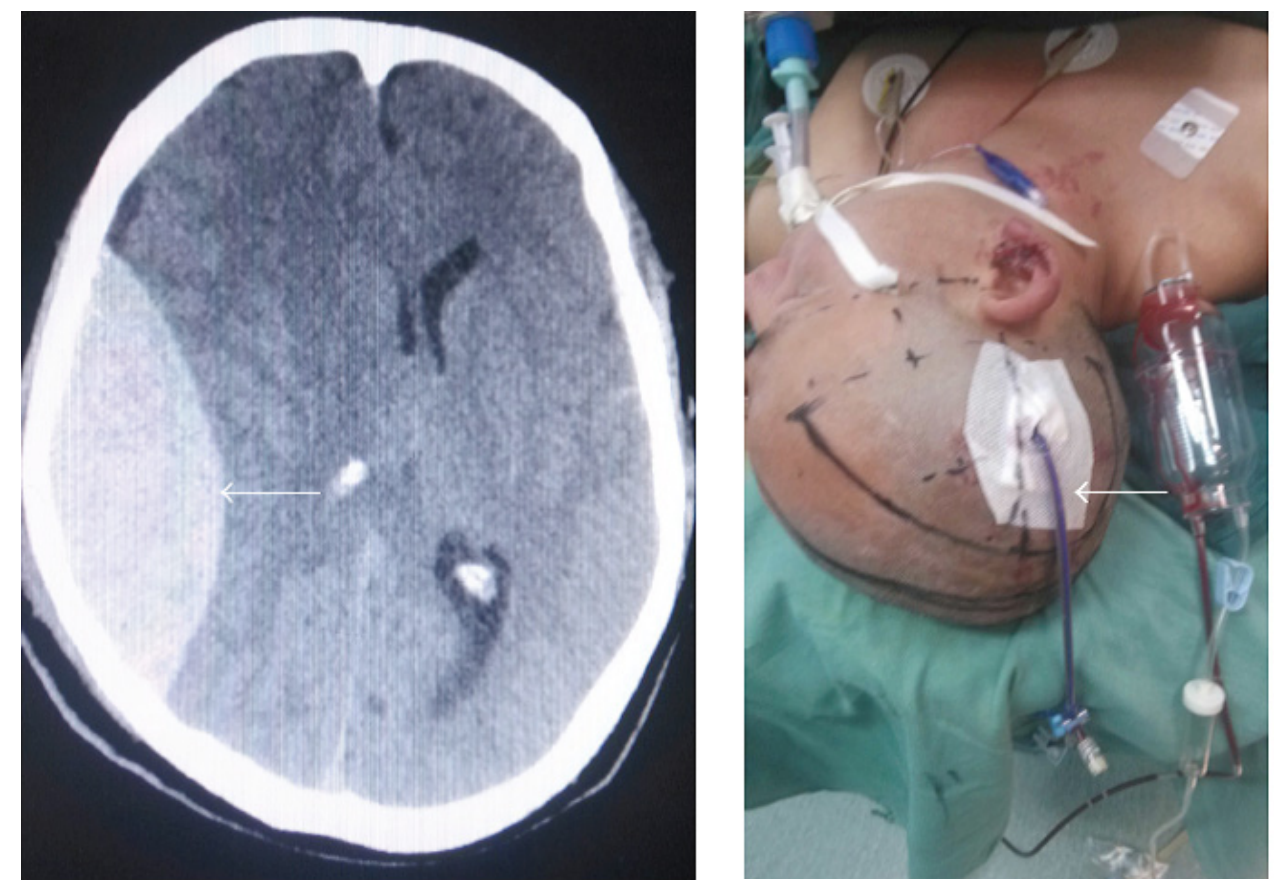

Figure 3 and 4. CT image of the head showing a large acute epidural hematoma with cerebral herniation in the right temporal-parietal region. Head CT scan image prior to surgery; hematoma volume was approximately $145 \mathrm{~mL}$ and the midline shift of $13 \mathrm{~mm}$. (b) Hematoma volume with rapid minimally invasive aspiration under local anesthesia was approximately $40 \mathrm{~mL}$; drainage tube was placed (Reference: Wang. 2016: 7). ${ }^{16}$ 
Minimally invasive surgery presents itself as one of the best alternatives to treat patients with EDH due to small incisions, reduced surgical time, reduced risk of anesthesia and avoid craniotomy. This procedure is used effectively in hemorrhages up to $50 \mathrm{ml}$ (WANG, 2016). ${ }^{16}$

Yao, Li and Sun $(2018)^{17}$ analyzed the case of one patient underwent surgical intervention within 80 hours to treat an epidural hematoma and achieved a nearly full recovery. These authors raise the hypothesis that exist a larger window for therapy in patients with epidural hematoma-induced paralysis. Moreover, Yao, Li and Sun (2018) ${ }^{17}$ describes the use of laminectomy, for decompression of spinal cord due the epidural hematoma, remains a viable treatment option, even if the diagnosis is established 8 hours after the onset of hematoma-induced symptoms.

Ren et al. (2018) $)^{4}$ raises the hypothesis that burr hole via tube and subsequent suction drainage could be an effective alternative treatment, that can avoid procedures more aggressive like open surgery with general anesthesia. However, these authors reinforce the necessity of more studies about this procedure to support the decision-making. On other hand, this procedure with suction drainage may have some disadvantages too, like: risk of infection, incomplete evacuation and prolonged hospital stays (REN et al., 2018). ${ }^{4}$

\section{Conclusion}

Traumatic extradural hematoma is rapid, sudden and potentially fatal, and therefore requires early diagnosis so that surgical intervention, which has been showing good results in the articles analyzed, prevents other preventable complications from occurring. Proper diagnosis and choice of the best surgical technique can be lifesaving, and it is necessary that the public health system, which receives many patients with this prognosis, has the proper structure, effective prevention strategies, and improved care for EDH.

The limitations found for this study were the large number of articles without open access and the difficulty of finding specific epidemiological data for EDH.

Regarding future research, it is suggested that they deepen the study on epidemiology and social factors related to EDH, and may bring new less invasive forms of treatment, both surgical and pharmacological. In addition, epidemiological data need to be increasingly large and reliable for better pathology compression.

\section{Acknowledgements}

The authors would like to thank the Academic League of Anatomy Oriented for the Surgical Clinic LAAOCCI for the opportunity to develop this research, and to thank UNIFACISA for providing full access to articles on the Up to Date platform.

\section{References}

1. Ahn ES, Proctor MR. Intracranial epidural hematoma in children: Epidemiology, anatomy and pathophysiology. Post TW, ed. UpToDate. Waltham, MA: UpToDate Inc. https://www.uptodate.com (Accessed on july 02, 2019.)

2. McBride W. Intracranial epidural hematoma in adults. Post TW, ed. UpToDate. Waltham, MA: UpToDate Inc. https://www.uptodate. com (Accessed on july 02, 2019.)

3. Health Sciences Descriptors: DeCS. *. rev. and enl. ed. São Paulo: BIREME / PAHO / WHO, 2017. Available from: <http://decs.bvsalud. org/l/homepagei.htm>. Access on 22 June 2017.

4. Ren H, Yin L, Ma L, Wei M, Ma X. Emergency bedside evacuation of a subset of large postoperative epidural hematomas after neurosurgical procedures. Med. 2018; 97:30

5. Verma SK, Borkar SA, Singh PK, et al. Traumatic posterior fossa extradural hematoma: Experience at level I trauma center. Asian J Neurosurg 2018;13:227-232

6. Ruschel LG, Rego FMM, Milano JB, Jung GS, Silva Jr LF, Ramina R. Spontaneous intracranial epidural hematoma during rivaroxaban treatment. Rev Assoc Med Bras 2016; 62 (8): 721-724

7. Ministério da Saúde. Departamento de Vigilância de Doenças e Agravos não Transmissíveis e Promoção da Saúde - DANTPS/SVS/ MS. http://svs.aids.gov.br/dantps/ (Accessed on october 10, 2018) 8. Chen CT, Lai HY, Chang TW, Lee CY. Repeated Spontaneous Intracranial Epidural Hemorrhage After Hysterical Crying. World Neurosurg. (2018) 114:34-36
9. Fang M, Zhou J, Yang D, et al. Management and outcomes of spinal epidural hematoma during vertebroplasty. Med 2018; 97:1-3 10. Li C, He R, Li X, Zhong Y, Ling L, Li F. Spontaneous spinal epidural hematoma mimicking transient ischemic attack. Med 2017; 96: 1-4 11. Ministério da Saúde. Sistema de Informações Hospitalares SIH/SUS. http://datasus.saude.gov.br (Accessed on october 10, 2018)

12. Rosi JrJ, Andrade AF, Yeng LC, et al. Epidural Hematoma: A Prospective Analysis of Morbidity and Mortality in 173 Patients. Arq Bras Neurocir 2015;34:20-24

13. Moreira MM, Adry RARC, Pereira CU. Characteristics of Contralateral Epidural Hematoma After Decompressive Craniectomy in Patients with Severe Traumatic Brain Injury. Systematic Review. J Bras Neurocirurg 2017; 28 (2): 101-110

14. Bulstrode H, Kabwama S, Durnford A, Hempenstall J, Chakraborty A. Temporising extradural haematoma by craniostomy using an intraosseous needle. Injury, Int. J. Care Injured 2017; 48:1098-1100 15. Peng A, Qi W, Cao D, et al. Fibrinolytic Therapy Improves Outcomes in Patients with Epidural Hematomas Following Cranioplasty: A Pilot Study. J Neurol Surg A Cent Eur Neurosurg 2018; 79(01): 039-044

16. Wang W. Minimally invasive surgical treatment of acute epidural hematoma: case series. Bio Med Research International 2016; 1-8 17. Yao YX, Li MX, Sun LJ. Good outcomes after the delayed removal of an epidural hematoma. A case report. Med 2018; 97:1-3 